\title{
Enumerating projective reflection groups
}

\author{
Riccardo Biagioli ${ }^{1}$ and Fabrizio Caselli ${ }^{2}$ \\ ${ }^{1}$ Université de Lyon, Université Lyon 1, Institut Camille Jordan, Villeurbanne, France \\ ${ }^{2}$ Dipartimento di matematica, Università di Bologna, Bologna, Italy
}

\begin{abstract}
Projective reflection groups have been recently defined by the second author. They include a special class of groups denoted $G(r, p, s, n)$ which contains all classical Weyl groups and more generally all the complex reflection groups of type $G(r, p, n)$. In this paper we define some statistics analogous to descent number and major index over the projective reflection groups $G(r, p, s, n)$, and we compute several generating functions concerning these parameters. Some aspects of the representation theory of $G(r, p, s, n)$, as distribution of one-dimensional characters and computation of Hilbert series of some invariant algebras, are also treated.

Résumé. Les groupes de réflexions projectifs ont été récemment définis par le deuxième auteur. Ils comprennent une classe spéciale de groupes notée $G(r, p, s, n)$, qui contient tous les groupes de Weyl classiques et plus généralement tous les groupes de réflexions complexes du type $G(r, p, n)$. Dans ce papier on définit des statistiques analogues au nombre de descentes et à l'indice majeur pour les groupes $G(r, p, s, n)$, et on calcule plusieurs fonctions génératrices. Certains aspects de la théorie des représentations de $G(r, p, s, n)$, comme la distribution des caractères linéaires et le calcul de la série de Hilbert de quelques algèbres d'invariants, sont aussi abordés.
\end{abstract}

Keywords: reflection groups, characters, permutation statistics, generating functions

\section{Introduction}

The study of permutation statistics, and in particular of those depending on up-down or descents patterns, is a very classical subject of study in algebraic combinatorics that goes back to the early 20th century to the work of MacMahon [Ma], and has found a new interest in the last decade after the fundamental work of Adin and Roichman [AR]. In their paper they defined a new statistic, the flag-major index that extend to the Weyl group of type $B$ the concept of major index, which is a classical and well studied statistic over the symmetric group. This paper opened the way to several others concerning the study of statistics over classical Weyl groups, wreath products, and some types of complex reflection groups. Now we have a picture of several combinatorial and algebraic properties holding for all these families of groups.

Recently, the second author introduced a new class of groups called projective reflection groups [Ca]. Among them, there is an infinite family denoted $G(r, p, s, n)$ where $r, p, s, n$ are integers such that $p, s \mid r$ and $p s \mid r n$. They include all complex reflection groups in fact $G(r, p, 1, n)=G(r, p, n)$. In this context is fundamental a notion of duality. Let $G=G(r, p, s, n)$, then its dual group is $G^{*}=G(r, s, p, n)$, where the parameters $p$ and $s$ are interchanged. In [Ca], it is shown that much of the theory of reflection groups can be extended to projective reflection groups, and that the combinatorics of the groups $G=G(r, p, s, n)$ is strictly related to the invariant theory of the dual group $G^{*}$. 
In this paper we continue that study. We introduce two descent numbers des and fdes, and a color sum col over $G(r, p, s, n)$, that together with the flag major index fmaj [Ca] allow the extension to $G(r, p, s, n)$ of the following results.

We show that the polynomial $\sum \chi(g) q^{\mathrm{fmaj}(g)}$, where $\chi$ denotes any one-dimensional character of $G(r, p, s, n)$ and the sum is over $g \in G(r, p, s, n)$, admits a nice product formula. This generalizes a classical result of Gessel and Simion [Wa] for the symmetric group, and the main results of Adin-GesselRoichman [AGR] for the Coxeter group of type $B$, and of the first author [Bi] for the type $D$ case.

The enumeration of permutations by number of descents and major index yields a remarkable $q$ analogue of a well-known identity for the Eulerian polynomials, usually called Carlitz's identity. This identity has been generalized in several ways and to several groups, see e.g. $[\overline{\mathrm{ABR}}],[\mathrm{BC}],[\overline{\mathrm{BB}}],[\mathrm{BZ}$, [BZ1], [ChG], [Re]. All these extensions, can be divided into two families, depending from the used descent statistic, i.e. geometric descents or flag descents. We give a general method to compute the trivariate distribution of des (or fdes), fmaj and col over $G(r, p, s, n)$. This unifies and generalizes all previous cited results. We further exploit our method to compute the generating function of the six statistics des, ides, fmaj, ifmaj, col, icol over the group $G(r, p, s, n)$, (ides $(g)$ denotes $\operatorname{des}\left(g^{-1}\right)$ and similarly for the others). From a specialization of our identity we deduce another important result, the computation of the generating function of the Hilbert series of the diagonal invariant algebras of the groups $G(r, p, s, n)$.

The definition of the above statistics depends on the particular order chosen on the colored integers numbers. By comparing our results with others in the literature we deduce that different choices of the order can or cannot give rise to same results. In some affirmative cases we provide bijective explanations of this phenomenon.

\section{Notation and preliminaries}

In this section we collect the notations that are used in this paper as well as the preliminary results that are needed.

We let $\mathbb{Z}$ be the set of integer numbers and $\mathbb{N}$ be the set of nonnegative integer numbers. For $a, b \in \mathbb{Z}$, with $a \leq b$ we let $[a, b]=\{a, a+1, \ldots, b\}$ and, for $n \in \mathbb{N}$ we let $[n] \stackrel{\text { def }}{=}[1, n]$. For $r \in \mathbb{N}$, we let $R_{r}: \mathbb{Z} \rightarrow[0, r-1]$ be the map "residue module $r$ ", where $R_{r}(i)$ is determined by $R_{r}(i) \equiv i \bmod r$. If $r \in \mathbb{N}, r>0$, we denote by $\zeta_{r}$ the primitive $r$-th root of unity $\zeta_{r} \stackrel{\text { def }}{=} e^{\frac{2 \pi i}{r}}$. As usual for $n \in \mathbb{N}$, we let $[n]_{q} \stackrel{\text { def }}{=} 1+q+q^{2}+\cdots+q^{n-1}$, and

$$
(a ; q)_{n} \stackrel{\text { def }}{=}\left\{\begin{array}{cc}
1, & \text { if } n=0 \\
(1-a)(1-a q) \cdots\left(1-a q^{n-1}\right), & \text { if } n \geq 1
\end{array}\right.
$$

Let $F \in \mathbb{C}\left[\left[q_{1}, q_{2}, \ldots\right]\right]$ be a formal power series with complex coefficients, and let $q_{i}$ be one of its variables. We denote by $\{F\}_{q_{i}^{p}}$ the power series obtained from $F$ by discarding all homogeneous components in the variable $q_{i}$ whose degree is not divisible by $p$.

The main subject of this work are the complex reflection groups [Sh], or simply reflection groups, with particular attention to their combinatorial representation theory. The most important example of a complex reflection group is the group of permutations of $[n]$, known as the symmetric group, that we denote by $S_{n}$. We know by the work of Shephard-Todd [ShT] that all but a finite number of irreducible reflection groups are the groups $G(r, p, n)$, where $r, p, n$ are positive integers with $p \mid r$, that we are going to describe. If $A$ is a matrix with complex entries, we denote by $|A|$ the real matrix whose entries are the 
absolute values of the entries of $A$. The wreath product $G(r, n)=G(r, 1, n)$ is given by all $n \times n$ matrices satisfying the following two conditions: the non-zero entries are $r$-th roots of unity; there is exactly one non-zero entry in every row and every column (i.e. $|A|$ is a permutation matrix). If $p$ divides $r$ then the reflection group $G(r, p, n)$ is the subgroup of $G(r, n)$ given by all matrices $A \in G(r, n)$ such that $\frac{\operatorname{det} A}{\operatorname{det}|A|}$ is a $\frac{r}{p}$-th root of unity.

A projective reflection group is a quotient of a reflection group by a scalar subgroup (see [Ca]). Observe that a scalar subgroup of $G(r, n)$ is necessarily a cyclic group $C_{s}$ of order $s$, generated by $\zeta_{s} I$, for some $s \mid r$, where $I$ denotes the identity matrix. It is also easy to characterize all possible scalar subgroups of the groups $G(r, p, n)$ : in fact the scalar matrix $\zeta_{s} I$ belongs to $G(r, p, n)$ if and only if $s \mid r$ and $p s \mid r n$. In this case we let $G(r, p, s, n) \stackrel{\text { def }}{=} G(r, p, n) / C_{s}$. If $G=G(r, p, s, n)$ then the projective reflection group $G^{*} \stackrel{\text { def }}{=} G(r, s, p, n)$, where the roles of the parameters $p$ and $s$ are interchanged, is always well-defined. We say that $G^{*}$ is the dual of $G$ and we refer the reader to [Ca] for the main properties of this duality.

We sometimes think of an element $g \in G(r, n)$ as a colored permutation. If the non-zero entry in the $i$-th row of $g \in G(r, n)$ is $\zeta_{r}^{c_{i}}$ we let $c_{i}(g) \stackrel{\text { def }}{=} R_{r}\left(c_{i}\right)$ and say that $c_{1}(g), \ldots, c_{n}(g)$ are the colors of $g$. Now it is easy to see that an element $g \in G(r, n)$ is uniquely determined by the permutation $|g| \in S_{n}$ defined by $|g|(i)=j$ if $g_{i, j} \neq 0$, and by its colors $c_{i}(g)$ for all $i \in[n]$. More precisely, when we consider an element of $G(r, n)$ as a colored permutation we represent its elements either as couples $\left(c_{1}, \ldots, c_{n} ; \sigma\right)$, where $\sigma=\sigma(1) \cdots \sigma(n)$ is a permutation in $S_{n}$ and $\left(c_{1}, \ldots, c_{n}\right)$ is the sequence of its colors, or in window notation as $g=[g(1), \ldots, g(n)]=\left[\sigma(1)^{c_{1}}, \ldots, \sigma(n)^{c_{n}}\right]$. Sometimes we will call $\sigma(i)$ the absolute value of $g(i)$, denoted $|g(i)|$. When it is not clear from the context, we will denote $c_{i}$ by $c_{i}(g)$. Moreover, if $c_{i}=0$, it will be omitted in the window notation of $g$. If we let $\operatorname{col}(g) \stackrel{\text { def }}{=} c_{1}(g)+\cdots+c_{n}(g)$, then for $p \mid r$ we can simply express the reflection group $G(r, p, n)$ by $G(r, p, n)=\left\{\left(c_{1}, \ldots, c_{n} ; \sigma\right) \in G(r, n) \mid \operatorname{col}(g) \equiv 0 \bmod p\right\}$, and for $q|r, p q| r n$ the projective reflection group by $G(r, p, s, n)=G(r, p, n) / C_{s}$, where $C_{s}$ is the cyclic subgroup of $G(r, p, n)$ of order $s$ generated by $(r / s, \ldots, r / s ; i d)$.

\section{Descent-type statistics}

In this section we recall the notions of descents, flag descents, and flag major index for the wreath products. Then we introduce analogous definitions for descents and flag descents for projective reflection groups. These two notions and the flag major index for $G(r, p, s, n)$ defined by Caselli in [Ca] will be used in the rest of the paper.

Definition 1 In all the paper we will use the following order, called color order

$$
1^{r-1}<\ldots<n^{r-1}<\ldots<1^{1}<\ldots<n^{1}<0<1<\ldots<n
$$

For $g \in G(r, n)$ we define the descent set as

$$
\operatorname{Des}_{G}(g) \stackrel{\text { def }}{=}\{i \in[0, n-1] \mid g(i)>g(i+1)\},
$$

where $g(0):=0$, and denote its cardinality by $\operatorname{des}_{G}(g)$. A geometric interpretation of this set in terms of Coxeter-like generators and length can be given as in [Ba]. If we consider only positive descents we 
obtain $\operatorname{Des}_{A}(g) \stackrel{\text { def }}{=} \operatorname{Des}_{G}(g) \backslash\{0\}$, and we denote by $\operatorname{des}_{A}(g)$ its cardinality. The flag major index [AR] and the flag descent number ([$[\overline{\mathrm{ABR}}],[\overline{\mathrm{BB}}])$ are defined by

$$
\operatorname{fmaj}(g) \stackrel{\text { def }}{=} r \cdot \operatorname{maj}(g)+\operatorname{col}(g) \text { and } \operatorname{fdes}(g) \stackrel{\text { def }}{=} r \cdot \operatorname{des}_{A}(g)+c_{1}(g)
$$

where as usual the major index $\operatorname{maj}(g)=\sum_{i \in \operatorname{Des}_{A}(g)} i$ is the sum of all positive descents of $g$.

Following [Ca, $\S 5]$, for $g=\left(c_{1}, \ldots, c_{n} ; \sigma\right) \in G(r, p, s, n)$ we let

$$
\begin{aligned}
\operatorname{HDes}(g) & \stackrel{\text { def }}{=}\left\{i \in[n-1] \mid c_{i}=c_{i+1}, \text { and } \sigma(i)>\sigma(i+1)\right\} \\
h_{i}(g) & \stackrel{\text { def }}{=} \#\{j \geq i \mid j \in \operatorname{HDes}(g)\} \\
k_{i}(g) & \stackrel{\text { def }}{=} \begin{cases}R_{r / s}\left(c_{n}\right) & \text { if } i=n \\
k_{i+1}+R_{r}\left(c_{i}-c_{i+1}\right) & \text { if } i \in[n-1] .\end{cases}
\end{aligned}
$$

We call the elements in $\operatorname{HDes}(g)$ the homogeneous descents of $g$. Note that $\left(k_{1}(g), \ldots, k_{n}(g)\right)$ is a partition such that $g=\left(R_{r}\left(k_{1}(g)\right), \ldots, R_{r}\left(k_{n}(g)\right) ; \sigma\right)$. Moreover it is characterized by the following property of minimality: if $\beta_{1} \geq \ldots \geq \beta_{n}$ with $\beta_{i} \equiv c_{i}$ for all $i \in[1, n]$, then $\beta_{i} \geq k_{i}(g)$, for all $i \in[1, n]$.

For $g=\left(c_{1}, \ldots, c_{n} ; \sigma\right) \in G(r, p, s, n)$, we let

$$
\lambda_{i}(g) \stackrel{\text { def }}{=} r \cdot h_{i}(g)+k_{i}(g) .
$$

The sequence $\lambda(g) \stackrel{\text { def }}{=}\left(\lambda_{1}(g), \ldots, \lambda_{n}(g)\right)$ is a partition such that $g=(\lambda(g) ; \sigma)$. The flag-major index for the projective reflection group $G(r, p, s, n)$ is defined in [Ca, $\$ 5$ ]

$$
\operatorname{fmaj}(g) \stackrel{\text { def }}{=}|\lambda(g)|=\sum_{i=1}^{n} \lambda_{i}(g) .
$$

We define the descent number and the flag descent number of $g \in G(r, p, s, n)$ respectively by

$$
\operatorname{des}(g) \stackrel{\text { def }}{=}\left\lfloor\frac{s \lambda_{1}(g)+r-s}{r}\right\rfloor \text { and } \operatorname{fdes}(g) \stackrel{\text { def }}{=} \lambda_{1}(g) .
$$

Finally, for $g \in G(r, p, s, n)$ we define the color of $g$ by

$$
\operatorname{col}(g) \stackrel{\text { def }}{=} \sum_{i=1}^{n} R_{r / s}\left(c_{i}(\tilde{g})\right),
$$

where $\tilde{g}$ is any lift of $g$ in $G(r, p, n)$.

Example 3.1 Let $g=(2,3,3,5,1,7,3,2 ; 27648153) \in G(6,2,3,8)$. Then we have $\operatorname{HDes}(g)=\{2,5\}$, $\left(h_{1}, \ldots, h_{8}\right)=(2,2,1,1,1,0,0,0)$ and $\left(k_{1}, \ldots, k_{8}\right)=(18,13,13,9,5,5,1,0)$, and so $\operatorname{des}(g)=15$, $\operatorname{fdes}(g)=6 \cdot 2+18=30, \operatorname{fmaj}(g)=6 \cdot 7+64=106$, and $\operatorname{col}(g)=6$.

Remark 1 From $[C a$ Lemma 5.1], it follows that for $p=s=1$, the flag major index on $G(r, p, s, n)$ defined in (5) coincides with the flag major index of Adin and Roichman for wreath products $G(r, n)$ 
defined in (3). Moreover the definition of fdes in (6) is consistent with that in (3), and $\operatorname{des}(g)=\operatorname{des}_{G}(g)$. To see this last equality notice that

$$
\operatorname{des}(g)=\left\lfloor\frac{\lambda_{1}(g)+r-1}{r}\right\rfloor=\operatorname{des}_{A}(g)+\left\lfloor\frac{c_{1}(g)+r-1}{r}\right\rfloor=\operatorname{des}_{G}(g),
$$

where the second equality holds since

$$
\left\lfloor\frac{c_{1}(g)+r-1}{r}\right\rfloor= \begin{cases}0, & \text { if } c_{1}(g)=0 ; \\ 1, & \text { otherwise. }\end{cases}
$$

These equalities give motivations to the definitions of the previous statistics.

\section{One-dimensional characters and flag major index}

The generating function of the major index with the unique non-trivial one-dimensional characters admits a nice factorization formula over the symmetric group, as shown in Theorem 4.1 by Gessel-Simion (see [Wa]). The same happens for the other classical Weyl groups, as proved by Adin-Gessel-Roichman [AGR] for the type $B$ case, and by the first author [Bi] for the type $D$ case. In this section we generalize these results to all projective groups of type $G(r, p, s, n)$.

We start by recalling the classical result of Gessel-Simion for the symmetric group. As usual for $\sigma \in S_{n}$, we denote by $\operatorname{inv}(\sigma) \stackrel{\text { def }}{=}\{(i, j) \in[n] \times[n] \mid i<j$ and $\sigma(i)>\sigma(j)\}$, the number of its inversions, and by $\operatorname{sign}(\sigma) \stackrel{\text { def }}{=}(-1)^{\operatorname{inv}(\sigma)}$ its sign.

Theorem 4.1 (Gessel-Simion) We have

$$
\sum_{\sigma \in S_{n}} \operatorname{sign}(\sigma) q^{\operatorname{maj}(\sigma)}=[1]_{q}[2]_{-q}[3]_{q} \cdots[n]_{(-1)^{n-1} q} .
$$

The particular case of the Weyl groups $B_{n} \stackrel{\text { def }}{=} G(2, n)$ is treated in [AGR]. In this paper the authors focus on the sum

$$
\sum_{g \in B_{n}}(-1)^{\ell(g)} q^{\mathrm{fmaj}(g)}
$$

where $\ell(g)$ is the Coxeter length of $g$ with respect to a given set of simple reflections, making use of a combinatorial interpretation of $\ell(g)$ due to Brenti $[\mathrm{BjB}]$. Theorem 4.2 is then achieved in this case since $(-1)^{\ell(g)}=(-1)^{\operatorname{inv}(|g|)}(-1)^{c(g)}$ for all $g \in B_{n}$. Although one can define an analogue of the Coxeter length for the wreath products $G(r, n)$, this does not lead to a one-dimensional character of the group, and the corresponding sum does not factorize nicely. This is why we focus on one-dimensional characters, obtaining in particular also a new proof in the case of Weyl groups of type $B$ that does not make use of the combinatorial interpretation of the length function.

The irreducible representations of $G(r, p, s, n)$ are classified in [Ca, §6]. In particular, the one-dimensional characters of $G(r, p, s, n)$ are all of the form

$$
\chi_{\epsilon, k}(g)=\epsilon^{\operatorname{inv}(|g|)} \zeta_{r}^{k \cdot c(g)},
$$

where $\epsilon= \pm 1$, and $k \in\left[0, \frac{r}{p}-1\right]$ with the further condition that $s$ divides $k n$, and $c(g)$ is the sum of the colors of any element in $G(r, p, n)$ representing the class of $g$ (in particular $c(g)=\operatorname{col}(g)$ if $s=1$ ). Our main result is the following one. 
Theorem 4.2 Let $\chi_{\epsilon, k}$ be a one dimensional character of $G(r, p, s, n)$. Then

$$
\sum_{g \in G(r, p, s, n)} \chi_{\epsilon, k}(g) q^{\mathrm{fmaj}(g)}=\left(\prod_{j \in[n-1]}\left[\frac{j r}{p}\right]_{\left(\epsilon^{j-1} \zeta^{k} q\right)^{p}}\right)\left[\frac{n r}{p s}\right]_{\left(\epsilon^{n-1} \zeta^{k} q\right)^{p}}\left\{[p]_{\zeta^{k} q}^{n-m}[p]_{\epsilon \zeta^{k} q}^{m}\right\}_{q^{p}},
$$

where $m=\left\lfloor\frac{n}{2}\right\rfloor$. (We recall that $\{F(q)\}_{q^{p}}$ is the polynomial obtained from $F(q)$ by discarding all the homogeneous components in the variable $q$ of degree not divisible by $p$.

For $r=2$ and $p=s=1$ in Theorem 4.2, we obtain [AGR, Theorems 5.1, 6.1, 6.2]; for $r=s=2$ and $p=1[$ Bi, Theorem 4.8].

\section{Carlitz's Identities}

In this section we give a general method to compute the trivariate distribution of des (or fdes), fmaj and col over $G(r, p, s, n)$. This will unify and generalize all results cited in the introduction and will provide two different generalizations of Carlitz's identity for the group $G(r, p, s, n)$.

For $f=\left(f_{1}, \ldots, f_{n}\right) \in \mathbb{N}^{n}$ let $|f|=f_{1}+\cdots+f_{n}$ and

$$
\mathbb{N}^{n}(p)=\left\{f \in \mathbb{N}^{n}|| f \mid \equiv 0 \bmod p\right\}
$$

Moreover, if $f \in \mathbb{N}^{n}(p)$ we let

$$
\operatorname{col}(f) \stackrel{\text { def }}{=} \sum_{i=1}^{n} R_{r / s}\left(f_{i}\right)
$$

be the sum of the residues of the $f_{i}$ 's modulo $r / s$.

Lemma 5.1 There is a bijection

$$
\mathbb{N}^{n}(p) \longleftrightarrow G(r, p, s, n) \times \mathcal{P}_{n} \times[0, s-1]
$$

such that if $f \leftrightarrow(g, \lambda, h)$, then

1. $|f|=\operatorname{fmaj}(g)+r|\lambda|+h n r / s$

2. $\operatorname{col}(f)=\operatorname{col}(g)$;

3. $\max f=\lambda_{1}(g)+r \lambda_{1}+h r / s$;

Proof: This result is similar to several others appearing in the literature and in particular is a special case of the bijection appearing in [Ca, Theorem 8.3] and so we simply describe how the bijection is defined for the reader's convenience. If $f=\left(f_{1}, \ldots, f_{n}\right) \in \mathbb{N}^{n}(p)$, then $g$ is the unique element in $G(r, p, s, n)$ having a lift $\tilde{g} \in G(r, p, n)$ satisfying:

- $f_{|g(i)|} \geq f_{|g(i+1)|}$ for all $i \in[n-1]$;

- if $f_{|g(i)|}=f_{|g(i+1)|}$ then $|g(i)|<|g(i+1)|$;

- $c_{i}(\tilde{g}) \equiv f_{|g(i)|} \bmod r$ for all $i \in[n]$. 
Letting $\mu$ be the partition obtained by reordering the entries in $\left(f_{1}, \ldots, f_{n}\right)$ in non-increasing order, one can show that $\mu-\lambda(g)$ (component-wise difference) is still a partition whose parts are all congruent to the same multiple of $r / s \bmod r$ (i.e. $\mu$ is $g$-compatible in the notation of [Ca]). The partition $\lambda \in \mathcal{P}_{n}$ and the integer $h \in[0, s-1]$ are therefore uniquely determined by the requirement $\mu=\lambda(g)+r \cdot \lambda+$ $(h r / s, \ldots, h r / s)$.

The inverse map of this bijection is much simpler. Let $(g, \lambda, h)$ be a triple in $G(r, p, s, n) \times \mathcal{P}_{n} \times[0, s-$ $1]$, then the corresponding element $f \in \mathbb{N}^{n}(p)$ is given by $f_{i}=\lambda_{\left|g^{-1}(i)\right|}(g)+r \lambda_{\left|g^{-1}\right|(i)}+h r / s$.

All the other statements are straightforward consequences, since $\lambda_{i}(g) \equiv c_{i}(\tilde{g}) \bmod r / s$ for any lift $\tilde{g}$ of $g$ and so we also have $\operatorname{col}(g)=\sum R_{r / s}\left(\lambda_{i}(g)\right)$.

We can now state the main results of this section.

Theorem 5.2

$$
\left\{\sum_{k \geq 0} t^{k}\left([k+1]_{q^{r / s}}+a q[k]_{q^{r / s}}\left[\frac{r}{s}-1\right]_{a q}\right)^{n}\right\}_{q^{p}}=\frac{\sum_{g \in G(r, p, s, n)} t^{\operatorname{des}(g)} q^{\mathrm{fmaj}(g)} a^{\operatorname{col}(g)}}{(1-t)\left(1-t^{s} q^{r}\right) \cdots\left(1-t^{s} q^{(n-1) r}\right)\left(1-t q^{n r / s}\right)}
$$

Letting $a=1$ in the previous result we easily obtain $[k+1]_{q^{r / s}}+q[k]_{q^{r / s}}\left[\frac{r}{s}-1\right]_{q}=\left[\frac{r}{s} k+1\right]_{q}$ and hence we obtain the following result.

Corollary 5.3 (Carlitz's identity for $G(r, p, s, n)$ with des)

$$
\left\{\sum_{k \geq 0} t^{k}\left[\frac{r}{s} k+1\right]_{q}^{n}\right\}_{q^{p}}=\frac{\sum_{g \in G(r, p, s, n)} t^{\operatorname{des}(g)} q^{\operatorname{fmaj}(g)}}{(1-t)\left(1-t^{s} q^{r}\right)\left(1-t^{s} q^{2 r}\right) \cdots\left(1-t^{s} q^{(n-1) r}\right)\left(1-t q^{n r / s}\right)} .
$$

The special case with $r=2, p=s=1$ of Corollary 5.3 is the main result of [ChG], and for $p=s=1$ we obtain [ChM, Theorem 10 (iv)].

A simple modification of the same ideas lead to the generalization of other identities that use flag descents.

\section{Theorem 5.4}

$$
\begin{aligned}
\sum_{k \geq 0} t^{k}\left(\left[Q_{r / s}(k)+1\right]_{q^{r / s}}\right. & \left.+a q[r / s-1]_{a q} \cdot\left[Q_{r / s}(k)\right]_{q^{r / s}}+a q^{m r / s+1)}\left[R_{r / s}(k)\right]_{a q}\right)^{n} \\
& =\frac{\sum_{g \in G(r, p, s, n)} t^{\mathrm{fdes}(g)} q^{\mathrm{fmaj}(g)} a^{\operatorname{col}(g)}}{(1-t)\left(1-t^{s} q^{r}\right) \cdots\left(1-t^{s} q^{(n-1) r / s}\right) \cdots\left(1-t q^{n r / s}\right)},
\end{aligned}
$$

where $Q_{r / s}(k)$ is the quotient of the division of $k$ by $r / s$.

By letting $a=1$ in Equation 8 , one obtain a second Carlitz's identity type, with flag-descents.

Theorem 5.5 (Carlitz's identity of $G(r, p, s, n)$ with fdes)

$$
\left\{\sum_{k \geq 0} t^{k}[k+1]_{q}^{n}\right\}_{q^{p}}=\frac{\sum_{g \in G(r, p, s, n)} t^{\mathrm{fdes}(g)} q^{\mathrm{fmaj}(g)}}{(1-t)\left(1-t^{r} q^{r}\right)\left(1-t^{r} q^{2 r}\right) \cdots\left(1-t^{r} q^{(n-1) r}\right)\left(1-t^{\frac{r}{s}} q^{\frac{n r}{s}}\right)} .
$$

For $r=2$ and $p=s=1$ we obtain [ABR, Theorem 4.2], for $r=s=2$ and $p=1$ [BC, Theorem 4.3], and for $p=1[\mathrm{BB}$, Theorem 11.2]. 


\section{Multivariate generating functions}

In this section we make further use of the bijection [Ca, Theorem 8.3] to compute new multivariate distributions for the groups $G(r, p, s, n)$. We first concentrate on the case of the groups $G(r, n)$ to make the used arguments more clear to the reader. We need to state the particular case of this bijection that is needed for our purposes. The bijection can be reformulated as follows in the special case of 2-partite partitions.

We recall that a 2-partite partition of length $n$ (see [GG]) is a $2 \times n$ matrix with non negative integer coefficients $f=\left(\begin{array}{cccc}f_{1}^{(1)} & f_{2}^{(1)} & \ldots & f_{n}^{(1)} \\ f_{1}^{(2)} & f_{2}^{(2)} & \ldots & f_{n}^{(2)}\end{array}\right)$ satisfying the following conditions:

- $f_{1}^{(1)} \geq f_{2}^{(1)} \geq \ldots \geq f_{n}^{(1)}$;

- If $f_{i}^{(1)}=f_{i+1}^{(1)}$ then $f_{i}^{(2)} \geq f_{i+1}^{(2)}$.

One may think of a 2-partite partition as a generic multiset of pairs of non negative integers (the columns of $f$ ) of cardinality $n$. If $f$ is a 2-partite partition we denote by $f^{(1)}$ and $f^{(2)}$ the first and the second row of $f$ respectively. We denote by $\mathcal{B}(n)$ the set of 2-partite partitions of length $n$ and we let

$$
\begin{aligned}
\mathcal{B}(r, n) & \stackrel{\text { def }}{=}\left\{f \in \mathcal{B}(n) \mid f_{i}^{(1)}+f_{i}^{(2)} \equiv 0 \bmod r \text { for all } i \in[n]\right\} \quad \text { and } \\
\mathcal{B}(r, s, 1, n) & \stackrel{\text { def }}{=}\left\{f \in \mathcal{B}(n) \mid \text { there exists } l \in[s-1]: f_{i}^{(1)}+f_{i}^{(2)} \equiv l r / s \bmod r \text { for all } i \in[n]\right\} .
\end{aligned}
$$

Note that $\mathcal{B}(1, n)=\mathcal{B}(n)$ and that $\mathcal{B}(r, n)=\mathcal{B}(r, 1,1, n)$.

Proposition 6.1 There exists a bijection between $\mathcal{B}(r, s, 1, n)$ and 5-tuples $(g, \lambda, \mu, h, k)$, where $g \in$ $G(r, 1, s, n), \lambda, \mu \in \mathcal{P}_{n}$ and $h, k \in[0, s-1]$. In this bijection if $f \leftrightarrow(g, \lambda, \mu, h, k)$ then

1. $\max \left(f^{(1)}\right)=\lambda_{1}(g)+r \lambda_{1}+h r / s$, and $\max \left(f^{(2)}\right)=\lambda_{1}\left(g^{-1}\right)+r \mu_{1}+k r / s$;

2. $\operatorname{col}\left(f^{(1)}\right)=\operatorname{col}(g)$, and $\operatorname{col}\left(f^{(2)}\right)=\operatorname{col}\left(g^{-1}\right)$;

3. $\left|f^{(1)}\right| \stackrel{\text { def }}{=} \sum f_{j}^{(1)}=\operatorname{fmaj}(g)+r|\lambda|+h n r / s$, and $\left|f^{(2)}\right| \stackrel{\text { def }}{=} \sum f_{j}^{(2)}=\operatorname{fmaj}\left(g^{-1}\right)+r|\mu|+k n r / s$.

Proof: This is again a particular case of [Ca, Theorem 8.3]. In this case the bijection is defined as follows: if $(g, \lambda, \mu, h, k) \leftrightarrow f$ then

$$
f_{i}^{(1)}=\lambda_{i}(g)+r \lambda_{i}+h r / s \text { and } f_{i}^{(2)}=\lambda_{|g(i)|}\left(g^{-1}\right)+r \mu_{|g(i)|}+k r / s,
$$

and all the other statements follow immediately.

We first state the result for the wreath products $G(r, n)$.

Theorem 6.2 Let $r \in \mathbb{N}$. Then

$$
\begin{aligned}
& \sum_{k_{1}, k_{2} \geq 0} t_{1}^{k_{1}} t_{2}^{k_{2}}\left(\prod_{\substack{i \in\left[0, r k_{1}\right], j \in\left[0, r k_{2}\right]: \\
i+j=0 \text { modr }}} \frac{1}{1-u a_{1}^{R_{r}(i)} a_{2}^{R_{r}(j)} q_{1}^{i} q_{2}^{j}}\right)= \\
& \quad=\sum_{n \geq 0} u^{n} \sum_{g \in G(r, n)} t_{1}^{\operatorname{des}(g)} t_{2}^{\operatorname{des}\left(g^{-1}\right)} q_{1}^{\mathrm{fmaj}(g)}, q_{2}^{\mathrm{fmaj}\left(g^{-1}\right)} a_{1}^{\operatorname{col}(g)} a_{2}^{\operatorname{col}\left(g^{-1}\right)} \prod_{j=0}^{n} \frac{1}{\left(1-t_{1} q_{1}^{j r}\right)\left(1-t_{2} q_{2}^{j r}\right)} .
\end{aligned}
$$


The corresponding result for the the full class of projective reflection group $G(r, p, s, n)$ is the following one (recall that $p \mid n \frac{r}{s}$ ).

Theorem 6.3 Let $r, p, s \in \mathbb{N}$, such that $s, p \mid r$. Then

$$
\begin{aligned}
\left\{\sum_{k_{1}, k_{2} \geq 0} t_{1}^{k_{1}} t_{2}^{k_{2}} \sum_{l=0}^{s-1}\left(\prod_{\substack{i \in\left[0, k_{1} \frac{r}{s}\right], j \in\left[0, k_{2} \frac{r}{s}\right]: \\
i+j=l \frac{r}{s} \bmod r}} \frac{1}{1-u a_{1}^{R_{r / s}(i)} a_{2}^{R_{r / s}(j)} q_{1}^{i} q_{2}^{j}}\right)\right\}_{q_{1}^{p}}= \\
=\sum_{n \geq 0} u^{n} \frac{\sum_{g \in G(r, p, s, n)} t_{1}^{\operatorname{des}(g)} t_{2}^{\operatorname{des}\left(g^{-1}\right)} q_{1}^{\operatorname{fmaj}(g)} q_{2}^{\mathrm{fmaj}\left(g^{-1}\right)} a_{1}^{\operatorname{col}(g)} a_{2}^{\operatorname{col}\left(g^{-1}\right)}}{\left(1-t_{1}\right)\left(1-t_{2}\right)\left(1-t_{1} q_{1}^{n \frac{r}{s}}\right)\left(1-t_{2} q_{2}^{n \frac{r}{s}}\right) \prod_{j=1}^{n-1}\left(1-t_{1}^{s} q_{1}^{j r}\right)\left(1-t_{2}^{s} q_{2}^{j r}\right)} .
\end{aligned}
$$

\subsection{Hilbert series of diagonal invariant algebras}

A specialization of Theorem 6.3 with $a_{1}=a_{2}=1$ yields the following result

$$
\left\{\sum_{l=0}^{s-1} \prod_{i+j \equiv l r / s} \frac{1}{1-u q_{1}^{i} q_{2}^{j}}\right\}_{q_{1}^{p}}=\sum_{n \geq 0} u^{n} \frac{\sum_{g \in G(r, p, s, n)} q_{1}^{\mathrm{fmaj}(g)} q_{2}^{\mathrm{fmaj}\left(g^{-1}\right)}}{\left(1-q_{1}^{n \frac{r}{s}}\right)\left(1-q_{2}^{n \frac{r}{s}}\right) \prod_{j=1}^{n-1}\left(1-q_{1}^{j r}\right)\left(1-q_{2}^{j r}\right)} .
$$

There is a nice algebraic interpretation of the previous identity. We let $S_{p}[X, Y]$ be the subalgebra of the algebra of polynomials in $2 n$ variables $x_{1}, \ldots, x_{n}, y_{1}, \ldots, y_{n}$ generated by 1 and the monomials whose degrees in both the $x$ 's and the $y$ 's variables are divisible by $p$. Then we can observe that the factor

$$
\frac{1}{\left(1-q_{1}^{n \frac{r}{s}}\right)\left(1-q_{2}^{n \frac{r}{s}}\right) \prod_{j=1}^{n-1}\left(1-q_{1}^{j r}\right)\left(1-q_{2}^{j r}\right)}
$$

is the bivariate Hilbert series of the invariant algebra corresponding to the "tensorial action" of the group $G(r, s, p, n)^{2}=G(r, s, p, n) \times G(r, s, p, n)$ (note the interchanging of the roles of $p$ and $s$ ) on the ring of polynomials $S_{p}[X, Y]$. Therefore, by [Ca, Corollary 8.6], we can deduce that

$$
\frac{\sum_{g \in G(r, p, s, n)} q_{1}^{\mathrm{fmaj}(g)} q_{2}^{\mathrm{fmaj}\left(g^{-1}\right)}}{\left(1-q_{1}^{n \frac{r}{s}}\right)\left(1-q_{2}^{n \frac{r}{s}}\right) \prod_{j=1}^{n-1}\left(1-q_{1}^{j r}\right)\left(1-q_{2}^{j r}\right)}=\operatorname{Hilb}\left(S_{p}[X, Y]^{\Delta G(r, s, p, n)}\right)
$$

where $\Delta G(r, s, p, n)$ is the diagonal embedding of $G(r, s, p, n)$ in $G(r, s, p, n)^{2}$. We can therefore conclude that Theorem 6.3 provides the following interpretation for the generating function of the Hilbert series of the diagonal invariant algebras of the groups $G(r, p, s, n)$.

Corollary 6.4 Let $G=G(r, p, s, n)$. Then we have

$$
\sum_{n \geq 0} u^{n} \operatorname{Hilb}\left(S_{s}[X, Y]^{\Delta G}\right)\left(q_{1}, q_{2}\right)=\left\{\sum_{l=0}^{p-1} \prod_{i+j \equiv l r / p} \frac{1}{1-u q_{1}^{i} q_{2}^{j}}\right\}_{q_{1}^{s}} .
$$




\section{Orderings}

In the literature, when considering statistics based on descents and/or inversions on the groups $G(r, n)$ at least two distinct orderings have been considered on the set of colored integers. In this last section we would like to clarify some of the relationships between two of these orderings concerning the results shown in this paper.

We recall that we have been using the order defined in (1). One can consider also the order $<^{\prime}$

$$
n^{r-1}<^{\prime} \cdots<^{\prime} n^{1}<^{\prime} \cdots<^{\prime} 1^{r-1}<^{\prime} \cdots<^{\prime} 1^{1}<^{\prime} 0<^{\prime} 1<^{\prime} \cdots<^{\prime} n .
$$

The order $<^{\prime}$ is the "good" one to give a combinatorial interpretation of the length function in $G(r, n)$ in terms of inversions (see e.g. [Ba], [BZ1]), while the order $<$ is often used in the study of some algebraic aspects such as the invariant theory of $B_{n}$ and $G(r, n)$ (see [AR], [Ca] $)$. Here we give a motivation for these choices. So we denote $\operatorname{Des}^{\prime}(g) \stackrel{\text { def }}{=}\left\{i \in[0, n-1] \mid g(i)>^{\prime} g(i+1)\right\}, \operatorname{des}^{\prime}(g) \stackrel{\text { def }}{=}\left|\operatorname{Des}^{\prime}(g)\right|$, and $\operatorname{fmaj}^{\prime}(g)=r \cdot \sum_{i \in \operatorname{Des}^{\prime}(g)} i+\operatorname{col}(g)$.

If one considers the special case of Theorem 5.2 with $p=s=1$, and relate it to [BZ, Theorem 5.1] one can deduce that the two polynomials

$$
\sum_{g \in G(r, n)} t^{\operatorname{des}(g)} q^{\mathrm{fmaj}(g)} a^{\operatorname{col}(g)} \text { and } \sum_{g \in G(r, n)} t^{\operatorname{des}^{\prime}(g)} q^{\mathrm{fmaj}^{\prime}(g)} a^{\operatorname{col}(g)}
$$

are equal. This can also be easily proved bijectively.

Proposition 7.1 There exists an explicit involution $\phi: G(r, n) \rightarrow G(r, n)$ such that

$$
\operatorname{des}(\phi(g))=\operatorname{des}^{\prime}(g) \text { and } \operatorname{col}(\phi(g))=\operatorname{col}(g),
$$

and in particular fmaj $(\phi(g))=$ fmaj' $^{\prime}(g)$.

Proof: For $g \in G(r, n)$ let $S(g)=\{g(1), \ldots, g(n)\}$. The set $S(g)$ is totally ordered by both $<$ and $<$. We let $\iota: S(g) \rightarrow S(g)$ be the unique involution such that $\iota(x)<\iota(y)$ if and only if $x<y$, for all $x, y \in S(g)$. We define $\phi(g)=[\iota(g(1)), \ldots, \iota(g(n))]$. It is clear that the map $\phi$ satisfies the conditions of the statement.

It follows from Proposition 7.1 that the two statistics fmaj and fmaj' are equidistributed over $G(r, n)$. Nevertheless the two polynomials $\sum_{g \in G(r, n)} \chi(g) q^{\mathrm{fmaj}(g)}$ and $\sum_{g \in G(r, n)} \chi(g) q^{\mathrm{fmaj}^{\prime}(g)}$, where $\chi$ is any linear character of $G(r, n)$, do not coincide in general. So, in order to obtain the results appearing in $\$ 4$ we must consider the order $<$. We should also mention that the polynomial $\sum_{g \in G(r, n)} \chi(g) q^{\mathrm{fmaj}^{\prime}(g)}$ does not factor nicely at all in general.

There is a more subtle difference if one considers the following multivariate distribution

$$
\sum_{g \in G(r, n)} t_{1}^{\operatorname{des}(g)} t_{2}^{\operatorname{des}\left(g^{-1}\right)} q_{1}^{\mathrm{fmaj}(g)} q_{2}^{\mathrm{fmaj}\left(g^{-1}\right)} a_{1}^{\operatorname{col}(g)} a_{2}^{\operatorname{col}\left(g^{-1}\right)},
$$

and the corresponding with the order $<^{\prime}$. In fact, by comparing Theorem 6.2 and [BZ1, Theorem 7.1] we deduce that these two polynomials coincide for $r=1,2$, and are distinct if $r>2$. The case $r=1$ being trivial, we can justify this coincidence for $r=2$ with a bijective proof. 
By the Robinson-Schensted correspondence for $B_{n}$ (see $\left.[\mathrm{StW}, \mathrm{Ca}]\right)$ we have a bijection

$$
g \mapsto\left[\left(P_{0}, P_{1}\right),\left(Q_{0}, Q_{1}\right)\right],
$$

where $\left(P_{0}, P_{1}\right)$ and $\left(Q_{0}, Q_{1}\right)$ are bitableaux of the same shape. Given a tableau $P$ let

$$
\operatorname{Des}(P)=\{i \mid \text { both } i \text { and } i+1 \text { belong to } P \text { with } i \text { strictly above } i+1\} \text {. }
$$

Let $\operatorname{Neg}(g)=\left\{i \in[n] \mid c_{i}(g)=1\right\}$. Let $\varphi: B_{n} \rightarrow B_{n}$ be the bijection defined by the requirement that if

$$
\begin{aligned}
g & \mapsto\left[\left(P_{0}, P_{1}\right),\left(Q_{0}, Q_{1}\right)\right], \quad \text { then } \\
\varphi(g) & \mapsto\left[\left(P_{0}, P_{1}^{\prime}\right),\left(Q_{0}, Q_{1}^{\prime}\right)\right],
\end{aligned}
$$

where $T^{\prime}$ denotes the transposed tableau. The next result gives a bijective proof of the equality between 9) and its $<^{\prime}$-analogue.

Proposition 7.2 The bijection $\varphi$ satisfies the following properties:

1. $\operatorname{Neg}(g)=\operatorname{Neg}(\varphi(g))$

2. $\operatorname{Des}(g)=\operatorname{Des}^{\prime}(\varphi(g))$.

3. $\operatorname{Des}\left(g^{-1}\right)=\operatorname{Des}^{\prime}\left(\varphi(g)^{-1}\right)$.

Example 7.3 If $g=[5,-2,-1,-4,6,-3,-7] \in B_{7}$, then

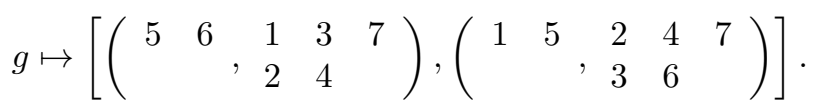

The element $\varphi(g)$ is the defined by

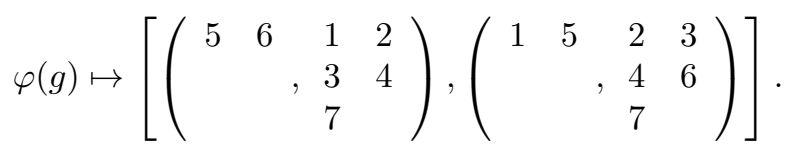

One then can check that $\varphi(g)=[5,-3,-7,-1,6,-4,-2]$. Hence $\operatorname{Neg}(g)=\operatorname{Neg}(\varphi(g))=\{2,3,4,6,7\}$, $\operatorname{Des}(g)=\operatorname{Des}^{\prime}(\varphi(g))=\{1,2,5\}$, and $\operatorname{Des}\left(g^{-1}\right)=\operatorname{Des}^{\prime}\left(\varphi(g)^{-1}\right)=\{0,1,3,6\}$.

\section{References}

[AR] R. M. Adin and Y. Roichman, The Flag Major Index and Group Actions on Polynomial Rings, Europ. J. Combinatorics, 22 (2001), 431-446.

[ABR] R. M. Adin, F. Brenti and Y. Roichman, Descent Numbers and Major Indices for the Hyperoctahedral Group, Adv. in Appl. Math., 27 (2001), 210-224.

[AGR] R. M. Adin, I. Gessel and Y. Roichman, Signed Mahonians, J. Combin. Theory Ser. A 109 (2005), no. $1,25-43$. 
[Ba] E. Bagno, Euler-Mahonian parameter on colored permutation groups, Sém. Loth. Combin., B51f (2004), 16 pp.

[BB] E. Bagno and R. Biagioli, Colored-Descent Representations of Complex Reflection Groups $G(r, p, n)$, Israel J. Math., 160 (2007), 317-348.

[Bi] R. Biagioli, Signed Mahonian polynomials for classical Weyl groups, Europ. J. Combin., 27 (2006), 207-217.

[BC] R. Biagioli and F. Caselli, Invariant algebras and major indices for classical Weyl groups, Proc. Lond. Math. Soc., 88 (2004), 603-631.

[BZ] R. Biagioli and J. Zeng, On some analogues of descent numbers and major index for the hyperoctahedral group, Sém. Loth. Combin., 61A (2010), article [B61Ak].

[BZ1] R. Biagioli and J. Zeng, Enumerating wreath products via Garsia-Gessel bijections, Europ. J. Combin., 32 (2011), 538-553.

[BjB] A. Björner and F. Brenti, Combinatorics of Coxeter Groups, G.T.M. 231, Springer-Verlag, New York, 2005.

[Ca] F. Caselli, Projective reflection groups, Israel J. Math., to appear, available at arXiv : 0902.0684.

[ChG] C.-O. Chow and I. M. Gessel, On the descent numbers and major indices for the hyperoctahedral group, Adv. in Appl. Math., 38 (2007), 275-301.

[ChM] C.-O. Chow and T. Mansour, A Carlitz identity for the wreath product $C_{r} 2 S_{n}$, Adv. in Appl. Math. (2011), to appear.

[GG] A. M. Garsia and I. Gessel, Permutation statistics and partitions, Adv. Math., 31 (1979), 288-305.

[Ma] P.A. MacMahon, Combinatorial Analysis, Chelsea, 1960. Originally published in two volumes by Cambridge Univ. Press, 1915-16.

[Sh] G. C. Shephard, Unitary groups generated by reflections, Canadian J. Math. 5 (1953), 364-383.

[ShT] G. C. Shephard and J. A. Todd, Finite unitary reflection groups, Canadian J. Math. 6 (1954), 274 304.

[StW] D.W. Stanton and D.E. White, A Schensted algorithm for rim hook tableaux, J. Combin. Theory Ser. A 40 (1985), 211-247.

[Re] V. Reiner, Signed permutation statistics, European J. Combin., 14 (1993), no. 6, 553-567.

[Re1] V. Reiner, Descents and one-dimensional characters for classical Weyl groups, Discrete Math., 140 (1995), 129-140.

[Wa] M. L. Wachs, An involution for signed Eulerian numbers, Discrete Math., 99 (1992), 59-62. 